\title{
¿Están correctamente anticoagulados nuestros pacientes con fibrilación auricular no valvular?
}

\author{
Joaquín Juan-Ortega*, Mireia Aguado-Furió, Ana Denia-Tomás, \\ Layla Abu-Omar-Peris, Anabel Esteve-Ardid y Artemio Pastor-Barberá
}

\author{
Centro de Salud Beniopa-Gandia, Valencia, España
}

Recibido el 13 de octubre de 2015; aceptado el 7 de junio de 2016

Disponible en Internet el 26 de julio de 2016

\section{PALABRAS CLAVE}

Fibrilación auricular; Anticoagulación; Atención primaria

\begin{abstract}
Resumen
Introducción: La fibrilación auricular es uno de los problemas de salud más importantes en el mundo por su elevada prevalencia, morbilidad y mortalidad, y porque es una causa importante de los ingresos hospitalarios y la principal indicación de la anticoagulación en atención primaria. Objetivo: El objetivo de nuestro estudio era definir el porcentaje de pacientes tratados, en la fibrilación auricular no valvular con anticoagulantes orales y las características clínicas de estos pacientes, calculando los riesgos embolígenos y hemorrágicos en este tipo de pacientes y su adecuación terapéutica.

Pacientes y método: Estudio descriptivo, transversal y retrospectivo, pacientes de un centro de salud, de tres cupos de Atención Primaria con diagnóstico de fibrilación auricular no valvular. Variables: datos demográficos, antecedentes personales (hipertensión arterial, diabetes mellitus, dislipidemia, alcoholismo, tabaquismo, cardiopatía isquémica, insuficiencia cardiaca, enfermedad cerebrovascular, insuficiencia renal, arteriopatía periférica y embolia sistémica), cálculo de las escalas CHA2DS2-VASc y HAS-BLED.

Resultados: Se encontraron 99 pacientes con fibrilación auricular no valvular de un total de 4.626 pacientes (2,15\%). Edad media $77 \pm 10$ años, $52,5 \%$ mujeres y $47,5 \%$ hombres. El $33 \%$ no toma ningún tratamiento anticoagulante, el $53 \%$ toma el acenocumarol y el resto (13\%) un nuevo anticoagulante oral (dabigatran, apixaban o rivaroxaban).

El 97\% de los pacientes presentan CHA2DS2-VASC $\geq 2$ (alto riesgo embolígeno), de los cuales el 30\% no está tomando ningún tratamiento anticoagulante. El $80 \%$ de los pacientes presentan HAS-BLED $\geq 3$ (alto riesgo hemorrágico), de los cuales el $55 \%$ toma el acenocumarol, presentando el $25 \%$ una razón internacional normalizada fuera de rango terapéutico.

Conclusiones: La fibrilación auricular no valvular es una de las principales causas del accidente vascular cerebral en nuestros pacientes. Se demuestra que el tratamiento anticoagulante en aquellos pacientes con alto riesgo embolígeno reduce los eventos cerebrovasculares en un 64\% y la mortalidad en un $26 \%$. En nuestro medio la mayoría de los pacientes diagnosticados con
\end{abstract}

\footnotetext{
* Autor para correspondencia.

Correo electrónico: ximojuan35@hotmail.es (J. Juan-Ortega).
} 
fibrilación auricular no valvular presentan ese riesgo, pero tenemos un $33 \%$ no anticoagulado y un $25 \%$ mal controlado con el acenocumarol (un total del $46 \%$ de los pacientes). Todo esto implica que casi el $50 \%$ de nuestros pacientes con fibrilación auricular no valvular presenten alto riesgo tromboembólico por falta de tratamiento o ineficacia de este.

(c) 2016 Sociedad Colombiana de Cardiología y Cirugía Cardiovascular. Publicado por Elsevier España, S.L.U. Este es un artículo Open Access bajo la licencia CC BY-NC-ND (http:// creativecommons.org/licenses/by-nc-nd/4.0/).

\section{KEYWORDS}

Atrial fibrillation; Anticoagulation; Primary care

\section{Introducción}

Entre las enfermedades crónicas, la fibrilación auricular (FA) es uno de los problemas de salud más importantes en el mundo por su elevada prevalencia (el 8,5\% en pacientes mayores de 65 años de edad) ${ }^{1}$, la morbilidad y la mortalidad, y porque es una causa importante de ingresos hospitalarios $^{2}$ y la principal indicación de anticoagulación en la atención primaria ${ }^{3}$. Además, no se debe olvidar que la tendencia de la población es a envejecer más, por lo que la importancia de la FA irá en aumento, más aún si se confirman los resultados de algunos estudios que indican que alrededor del $20 \%$ de los pacientes $>60$ años de edad, presentan la FA y no están diagnosticados ${ }^{4}$.
El riesgo de la enfermedad cerebrovascular asociado a la FA, principal causa de la mortalidad y la morbilidad, es variable en función de la coexistencia de otros factores de riesgo. En la práctica clínica se puede estimar este riesgo empleando escalas como CHA2DS2-VASc, de tal forma que puntuaciones $\geq 2$ en un paciente con la FA hacen necesaria la anticoagulación ${ }^{5}$. Se ha demostrado que la anticoagulación con fármacos antagonistas de la vitamina K (AVK) reduce la incidencia de los eventos tromboembólicos en un $64 \%$ y la mortalidad en un $26 \%$. No obstante, la anticoagulación con AVK tiene importantes limitaciones; la más importante es la variabilidad de la respuesta clínica, ya que la razón internacional normalizada (INR) debe mantenerse entre 2 y 3 para asegurar el efecto anticoagulante de estos 
fármacos ${ }^{6}$, múltiples factores ambientales (dieta, fármacos, etc.) y genéticos influyen en el efecto terapéutico y con frecuencia hacen que la INR se encuentre fuera del rango establecido, lo que expone a los pacientes a eventos trombóticos y hemorrágicos. La comercialización en los últimos años de nuevos fármacos anticoagulantes - con menos interacciones farmacológicas y ambientales, rango terapéutico más amplio, demostrada eficacia y seguridad, al menos similar, a los AVK y coste/eficaces en paciente con la INR fuera de rango- ha hecho que las autoridades sanitarias los recomienden para este grupo de pacientes, en especial cuando existe un riesgo hemorrágico. En los pacientes con la fibrilación auricular no valvular, una vez se ha establecido la indicación del tratamiento con anticoagulantes orales según el riesgo embolígeno moderado-alto con CHA2DS2-VASc se debe calcular el riesgo hemorrágico con la tabla HAS-BLED. Una puntuación HAS-BLED $\geq 3$ indica «riesgo elevado» de sangrado con dicumarínicos, por lo que se debe emplear con extrema precaución o valorar la posibilidad de otras alternativas terapéuticas, como los nuevos anticoagulantes.

El objetivo de nuestro estudio era definir el porcentaje de pacientes tratados, en fibrilación auricular no valvular (FANV), con anticoagulantes orales y las características clínicas de estos pacientes, calculando los riesgos embolígenos y hemorrágicos en estos tipos de pacientes y su adecuación terapéutica.

\section{Pacientes y método}

Estudio descriptivo, transversal y retrospectivo en un centro de salud urbano de Gandía, en la provincia de Valencia (España), de los pacientes de tres cupos de Atención Primaria con diagnóstico de la FANV. Se entiende por cupo como el número de pacientes asignados a cada médico del Centro de Atención Primaria, siendo en nuestro país de 1.500-1.600 pacientes de media por cada profesional. Se excluyen aquellos pacientes de ese listado que presenten menos de un año del diagnóstico.

Se registraron datos demográficos (edad, sexo), antecedentes personales (hipertensión arterial, diabetes mellitus, dislipidemia, alcoholismo, tabaquismo, cardiopatía isquémica, insuficiencia cardiaca, enfermedad cerebrovascular, insuficiencia renal, arteriopatía periférica y embolia sistémica), datos clínicos actuales (peso, talla, los controles de la INR del último año -entendiendo como la INR fuera de rango si presenta el $40 \%$ de estos inferiores a 2 o superiores a 3, cifras de presión arterial sistólica y diastólica, glucohemoglobina, tasa de filtrado glomerular, colesterol total, colesterol unido a lipoproteínas de baja densidad, colesterol unido a lipoproteínas de alta densidad, triglicéridos, GOT, GPT, No. de los fármacos) y tipo de anticoagulante empleado (acenocumarol o NACO). Disponer de estas variables permitió el cálculo de las escalas CHA2DS2-VASc y HAS-BLED (hipertensión, función renal/hepática anormal, ictus, antecedentes de hemorragia o predisposición a ella, labilidad de la razón internacional normalizada, edad $>65$ años de edad y toma concomitante de fármacos o alcohol), cuyos puntos de corte son $\geq 2$ la primera $y \geq 3$ la última. Se clasifica como obesos a los pacientes con índice de masa corporal (IMC) $\geq 30$. Se definió al paciente como hipertenso, diabético o dislipidémico cuando estaba diagnosticado como tal en la
Tabla 1 Características demográficas de los pacientes

\begin{tabular}{ll}
\hline Edad & $77 \pm 10$ años \\
Sexo & \\
Hombre & $47,5 \%$ \\
Mujer & $52,5 \%$ \\
Otras patologías asociadas: & \\
HTA & $69 \%$ \\
Dislipidemia & $49 \%$ \\
Diabetes & $38 \%$ \\
Cardiopatia isquémica & $41 \%$ \\
AVC & $26 \%$ \\
Hemorragias previas & $15 \%$ \\
Consumo de fármacos & \\
$\leq 3$ & $30 \%$ \\
$\geq 4$ & $70 \%$ \\
\hline
\end{tabular}

historia clínica electrónica, o estaba tomando medicación. Se registra el antecedente de la enfermedad cardiovascular (cardiopatía isquémica, insuficiencia cardiaca, enfermedad cerebrovascular o arteriopatía periférica) cuando tengan registrado en la historia clínica electrónica el antecedente de ingreso o asistencia en urgencias por alguno de los procesos.

El análisis estadístico se realizó mediante el programa SPSS-19. Se utilizaron estimadores descriptivos (proporciones en las variables categóricas, y medianas, máximos y mínimos o medias y desviación estándar en las variables cuantitativas).

\section{Resultados}

Se analizaron un total de 99 pacientes diagnosticados con la fibrilación auricular no valvular de un total de 4.626 pacientes que forman los 3 cupos de Atención Primaria $(2,15 \%)$. La edad media es de $77 \pm 10$ años siendo el $52,5 \%$ mujeres frente a un $47,5 \%$ de hombres. Un $69 \%$ presenta asociado HTA, un $49 \%$ dislipidemia y un $38 \%$ diabetes. Antecedentes de cardiopatía isquémica lo presentan $41 \%$, de AVC el $26 \%$ y algún episodio hemorrágico lo presentan el $15 \%$.

La mayoría de los pacientes están polimedicados, consumiendo el $70 \%$ más de 4 fármacos (tabla 1 ). El 33\% no toma ningún tratamiento anticoagulante, el 53\% toma el acenocumarol y el resto (13\%) un NACO (dabigatran, apixaban o rivaroxaban).

De los pacientes que toman el acenocumarol, el $25 \%$ no tienen la INR en rango.

El 97\% de los pacientes presentan CHA2DS2-VASC $\geq 2$ (alto riesgo embolígeno), de los cuales el $30 \%$ no está tomando ningún tratamiento anticoagulante (fig. 1). El $80 \%$ de los pacientes presentan HAS-BLED $\geq 3$ (alto riesgo hemorrágico), de los cuales el 55\% toma el acenocumarol (fig. 2), presentando el $25 \%$ un INR fuera de rango terapéutico.

\section{Discusión}

La FA es la arritmia cardiaca más frecuente en la práctica clínica diaria y se asocia a un marcado aumento del riesgo de accidentes tromboembólicos. Está ampliamente 


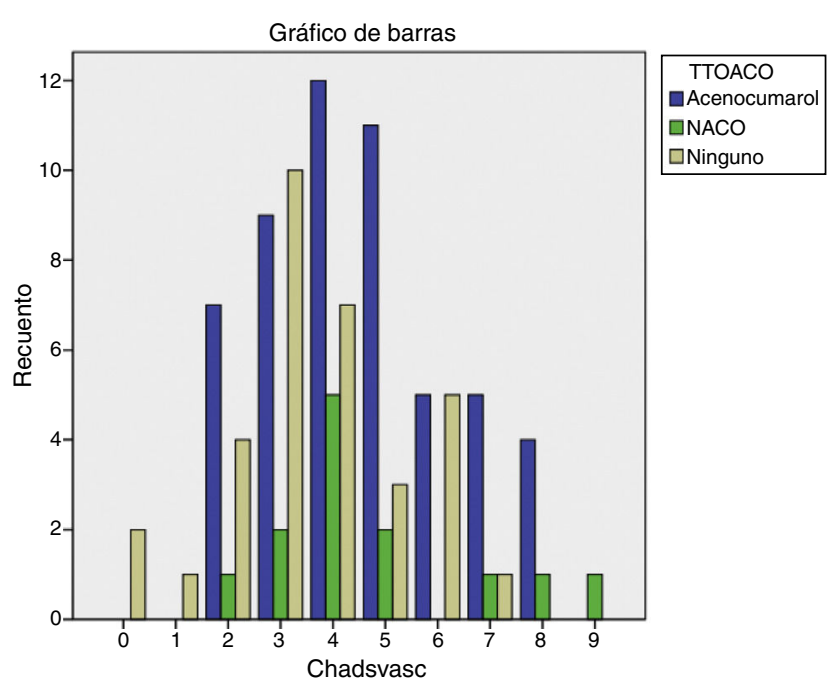

Figura 1 CHA2DS2-VASc en pacientes con la FANV y tratamiento anticoagulante.

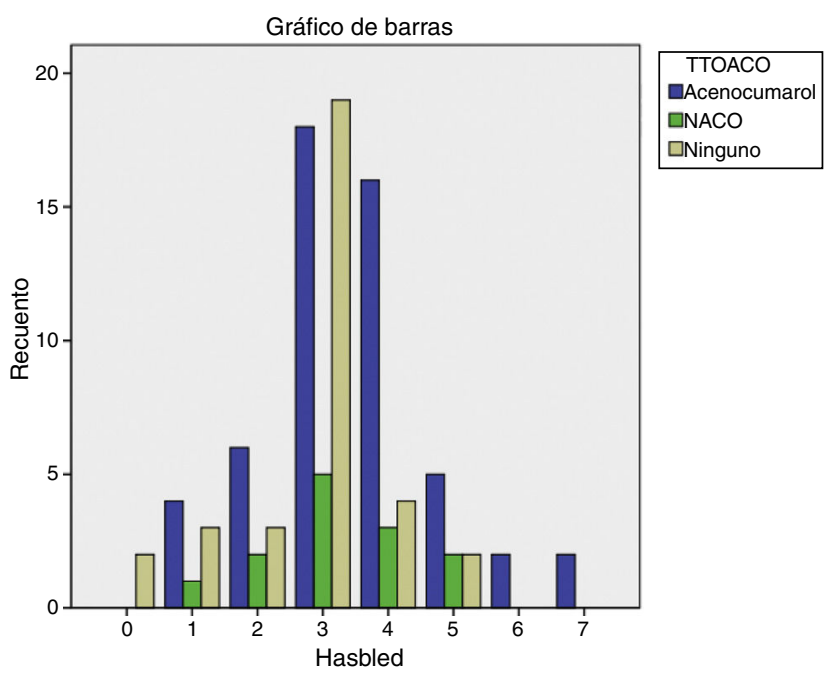

Figura 2 HAS-BLED en pacientes con la FANV y tratamiento anticoagulante.

demostrado que el tratamiento antitrombótico con anticoagulantes orales se asocia a un importante descenso del riesgo de accidentes tromboembólicos, pero puede ocasionar un incremento significativo del riesgo de complicaciones hemorrágicas mayores. Por eso, antes de indicar el tratamiento anticoagulante en un paciente con la FA, resulta imprescindible hacer una adecuada valoración del beneficio/riesgo del tratamiento, utilizando los métodos de estratificación de riesgo de embolia (CHA2DS2-VASc) y de sangrado (HAS-BLED) según las recomendaciones de la ESC, eligiendo el fármaco (acenocumarol o NACO) más adecuado para ello.

La fibrilación auricular no valvular es una de las principales causas del accidente vascular cerebral en nuestros pacientes. Demostrado queda que el tratamiento anticoagulante en aquellos pacientes con alto riesgo embolígeno reduce los eventos cerebrovasculares en un $64 \%$ y la mortalidad en un $26 \%$. En nuestro medio la mayoría de los pacientes diagnosticados con la FANV presentan ese riesgo, pero tenemos un $33 \%$ no anticoagulado y un $25 \%$ mal controlado con el acenocumarol (un total del $46 \%$ de los pacientes). Todo esto implica que casi el $50 \%$ de nuestros pacientes con la FANV presenten alto riesgo tromboembólico por falta de tratamiento o ineficacia de éste. Así mismo, 4 de cada 5 de nuestros pacientes con la FANV presentan un alto riesgo hemorrágico, medicándose el $50 \%$ de estos con el acenocumarol, en detrimento de los NACO que podrían disminuir considerablemente este riesgo.

A la vista de los resultados, podemos concluir que anticoagulamos a pocos pacientes con la fibrilación auricular no valvular.

\section{Responsabilidades éticas}

Protección de personas y animales. Los autores declaran que para esta investigación no se han realizado experimentos en seres humanos ni en animales.

Confidencialidad de los datos. Los autores declaran que en este artículo no aparecen datos de pacientes.

Derecho a la privacidad y consentimiento informado. Los autores declaran que en este artículo no aparecen datos de pacientes.

\section{Conflicto de intereses}

Los autores declaran no tener ningún conflicto de intereses.

\section{Bibliografía}

1. Barrios V, Calderón A, Escobar C, De la Figuera M. Pacientes con fibrilación auricular asistidos en consultas de atención primaria. Estudio Val-FAAP. Rev Esp Cardiol. 2012;65:47-53.

2. Wattigney WA, Mensah GA, Croft JB. Increasing trends in hospitalization for atrial fibrillation in the United States, 1985 through 1999: implications for primary prevention. Circulation. 2003;108:711-6.

3. Hirsch J, Fuster V, Ansell J, Halperin JL. American Heart Association/American College of Cardiology Foundation guide to warfarin therapy. Circulation. 2003;107:1692-711.

4. Clua-Espuny JL, Lechuga-Duran I, Bosch-Princep R, Roso-Llorach A, Panisello-Tafalla A, Lucas-Noll J, et al. Prevalencia de la fibrilación auricular desconocida y la no tratada con anticoagulantes. Estudio AFABE. Rev Esp Cardiol. 2013;66:545-52.

5. Camm AJ, Kirchhof P, Lip GY, Schotten U, Savelieva I, Ernst S, et al. European Heart Rhythm Association; European Association for Cardio-Thoracic Surgery, Guidelines for the management of atrial fibrillation: the Task Force for the Management of Atrial Fibrillation of the European Society of Cardiology (ESC). Eur Heart J. 2010;31:2369-429.

6. Fuster V, Rydén LE, Cannom DS, Crijns HJ, Curtis AB, Ellenbogen KA, et al. 2011 ACCF/AHA/HRS focused updates incorporated into the ACC/AHA/ESC 2006 Guidelines for the management of patients with atrial fibrillation: a report of the American College of Cardiology Foundation/American Heart Association Task Force on Practice Guidelines developed in partnership with the European Society of Cardiology and in collaboration with the European Heart Rhythm Association and the Heart Rhythm Society. J Am Coll Cardiol. 2011;57:e101-98. 